\title{
Unraveling Brain Functional Connectivity of encoding and retrieval in the context of education
}

\author{
Rajanikant Panda $^{\mathrm{a}, \mathrm{c}}$, Rose Dawn Bharath ${ }^{\mathrm{a}, \mathrm{c}, *}$, Lija George ${ }^{\mathrm{a}, \mathrm{c}}$, Silpa Kanungo ${ }^{\mathrm{a}, \mathrm{c}}$, Rajakumari P. Reddy ${ }^{\mathrm{b}, \mathrm{c}}$, \\ Neeraj Upadhyay $^{\mathrm{a}, \mathrm{c}}$, Arumugam Thamodharan ${ }^{\mathrm{a}, \mathrm{c}}$, Jamuna Rajeshwaran ${ }^{\mathrm{b}, \mathrm{c}}$, Shobini L. Rao ${ }^{\mathrm{b}, \mathrm{c}}$, \\ Arun Kumar Gupta ${ }^{\text {a,c }}$ \\ ${ }^{a}$ Dept. of Neuroimaging and Interventional Radiology, NIMHANS, India \\ ${ }^{\mathrm{b}}$ Dept. of Clinical Psychology, NIMHANS, India \\ ${ }^{\mathrm{c}}$ Centre for Cognitive Neuroscience, Advanced Brain Imaging Facility, National Institute of Mental Health and Neuro Science (NIMHANS), Bangalore, India
}

\section{A R T I C L E I N F O}

Article history:

Accepted 30 January 2014

Keywords:

Functional connectivity MRI

Seed based functional correlation

School educated and college educated

Cognitive reserve

\begin{abstract}
A B S T R A C T
Human memory is an enigmatic component of cognition which many researchers have attempted to comprehend. Accumulating studies on functional connectivity see brain as a complex dynamic unit with positively and negatively correlated networks in perfect coherence during a task. We aimed to examine coherence of network connectivity during visual memory encoding and retrieval in the context of education. School Educated (SE) and College Educated (CE) healthy volunteers $(n=60)$ were recruited and assessed for visual encoding and retrieval. Functional connectivity using seed to voxel based connectivity analysis of the posterior cingulate cortex (PCC) was evaluated. We noticed that there were reciprocal dynamic changes in both dorsolateral prefrontal cortex (DLPFC) region and PCC regions during working memory encoding and retrieval. In agreement with the previous studies, there were more positively correlated regions during retrieval compared to encoding. The default mode network (DMN) networks showed greater negative correlations during more attentive task of visual encoding. In tune with the recent studies on cognitive reserve we also found that number of years of education was a significant factor influencing working memory connectivity. SE had higher positive correlation to DLPFC region and lower negative correlation to DMN in comparison with CE during encoding and retrieval.
\end{abstract}

(C) 2014 Elsevier Inc. All rights reserved.

\section{Introduction}

The importance of memory cannot be understated since our very survival depends on our ability to remember who we are, who others are, our past experiences, what is dangerous, what is safe, etc. Atkinson-Shiffrin model (Bjork \& Whitten, 1974) remains the most popular model for studying memory which describes memory as a sequence of three stages, from sensory to short-term to long term memory, rather than as a unitary process. All aspects of memory, can be viewed as the activation of network memory, that is, the increased firing of the cortical neurons making up a memory network (Joaquín M. Fuster, 1997). Networks remain open-ended throughout life, subject to expansion and recombination by new experience. The networks of perceptual and motor memory appear hierarchically organized on a foundation of phyletic memory-that

\footnotetext{
* Corresponding author at: Centre for Cognitive Neuroscience, Advanced Brain Imaging Facility, National Institute of Mental Health and Neuro Science (NIMHANS), Hosur Road, Bangalore 560029, India. Fax: +91 08026564830.

E-mail addresses: cns.researchers@gmail.com, drrosedawn@yahoo.com (R.D. Bharath).
}

is, primary sensory and motor cortex (Joaquín M. Fuster, 1997). Short-term memory is represented in a network involving the prefrontal and posterior sensory cortices. The prefrontal cortex controls subsidiary posterior cortices, enabling retention of shortlived information relevant to ongoing goal-directed behavior. Long-term declarative memory is also represented in the cerebral cortex in a domain-specific fashion. For instance, in the inferior temporal cortex, which is involved in object recognition, some neurons encode stimulus repetition, while others learn to encode an elemental semantic-like association between visual images. (T. Fukushima, 2003). The process of encoding varies with the levels of shallow and deep processing (Craik \& Tulving, 1975). The retrieval is influenced by cues, encoding specificity, state-dependent learning and transfer-appropriate processing. Neurobiology of memory involves connection, cognition, compartmentalization, and consolidation (Milner, Squire, and Kandel, 1998; Eichenbaum, 2011).

With the developments in the field of functional MRI (fMRI) a very high level of integrative real time understanding of working memory is now possible as we are able to see brain in real time as 
functionally defined set of networks that transiently interact to perform a particular neural function. Accumulating studies on functional connectivity now see brain as a complex dynamic unit with positively and negatively correlated networks in perfect coherence during a task (Chanraud, Pitel, Pfefferbaum, \& Sullivan, 2011; Esposito et al., 2009; Fox, Zhang, Snyder, \& Raichle, 2009; Hampson, Driesen, Skudlarski, Gore, \& Constable, 2006; Mayer, Roebroeck, Maurer, \& Linden, 2010; Meda, Stevens, Folley, Calhoun, \& Pearlson, 2009; Sala-Llonch et al., 2012; Van den Bosch et al., 2012). Taskpositive networks are regions that are simultaneously activated during active cognitive processing. Frontoparietal network is a prominent task positive working memory network (Champod \& Petrides, 2010; Deiber et al., 1997; Iidaka, Matsumoto, Nogawa, Yamamoto, \& Sadato, 2006; LaBar, Gitelman, Parrish, \& Mesulam, 1999). Dorsolateral prefrontal cortex is considered as hub for many networks especially in the context memory and education (Champod \& Petrides, 2007; Collette et al., 1999; Gerton et al., 2004; Sun et al., 2005). Task-negative networks (resting-related networks) are those which are active during passive or stimulus-independent thought, and are subsequently deactivated during active processing. One of the well documented resting related networks is DMN. This network comprises a set of highly functionally connected regions such as medial prefrontal cortex (MPFC), posterior cingulate cortex (PCC), lateral and medial temporal lobes and posterior inferior parietal lobule (Greicius, Supekar, Menon, \& Dougherty, 2009; Raichle et al., 2001; Uddin, Clare Kelly, Biswal, Castellanos, \& Milham, 2009; Fransson et al., 2005; Damoiseaux et al., 2006; Fox and Raichle, 2007; Buckner, Andrews-Hanna, and Schacter, 2008; Johnson et al., 2012). The negative correlation between regions in the DMN during a cognitive task suggests that the circuit interferes with or distracts from cognitive processing and facilitates cognitive performance. In prior studies, a negative correlation between working memory performance and task-irrelevant mental processing was reported (Chanraud et al., 2011; Esposito et al., 2009; Fox et al., 2005; Fox et al., 2009; Hampson et al., 2006; Mayer et al., 2010; Meda et al., 2009; Michels et al., 2010; Sala-Llonch et al., 2012; Van den Bosch et al., 2012). A recent study observed and found that not only there is negative correlation between the DMN and cognitive tasks but the degree is proportionally related with the demands of the cognitive function (Sala-Llonch et al., 2012). Further study confirmed that the relative extent of the anterior and posterior midline spots within the DMN was negatively correlated in the PCC with the level of task difficulty. The study concluded that the working-memory function is related to a spatial re-configuration of the DMN functional connectivity which could function as a novel predictor of the working-memory efficiency (Esposito et al., 2009). From the perspective of development, Van den Bosch et al. studied the brain connectivity during verbal working memory in children and adolescents. They noted children have higher task-related connectivity at lower loads, but they tend to equalize with the adolescents with higher loads and non-load related network involving the orbital frontal and anterior cingulate cortices showed less connectivity in children (Van den Bosch et al., 2012). In a study done by Champod and Petrides (2007), it was noted that the PCC is centrally involved in manipulation processes, whereas activation of the DLFC is related to the monitoring of the information that is being manipulated and their relative contribution to working memory. Brain deactivation patterns during working memory and visual attention tasks was noted in task fMRI (Tomasi, Ernst, Caparelli, \& Chang, 2006). Mayer et al. noted task induced brain deactivation in DMN region in visual working memory and attention task. They reported task-induced deactivations within regions of the DMN with a segregation of areas that were additively deactivated by an increase in the demands on both attention and WM. Attention selective deactivations appeared in the left ventrolateral and medial prefrontal cortex and the left lateral temporal cortex. Conversely, WM selective deactivations were reported in the right hemisphere including the medial-parietal, the lateral temporo-parietal, and the medial prefrontal cortex. Moreover they also reported, during WM encoding deactivated regions showed task specific functional connectivity. Their findings demonstrate that task induced deactivations within parts of the DMN depend on the specific characteristics of the cognitive process (Mayer et al., 2010). Recently an EEG-fMRI study found that low frequency band (theta and alpha) activity negatively correlated with the BOLD signal during the retention phase of a WM task. They found negative BOLD signal correlations with lower frequency of EEG (theta band) in the MPFC, posterior parietal cortex (PPC), and cingulate cortex (ACC and PCC). For alpha1, positive correlations with the BOLD signal were found in ACC, MPFC, and PCC; negative correlations were observed in DLPFC, PPC, and inferior frontal gyrus (IFG). Beta and gamma showed positive correlations with BOLD in DLPFC, MPFC and IFG (Michels et al., 2010).

Connectivity analysis could be either task independent (resting state) based connectivity or task based functional connectivity. Task based functional connectivity could be superior in understanding dynamic changes involved in network in relation to a task (Greicius, Krasnow, Reiss, \& Menon, 2003; Mayer et al., 2010; Wang, Bartolotti, Amaral, \& Booth, 2013).

Cognitive reserve (CR) hypothesis emphasizes inter-individual differences in the effective recruitment of neural networks and cognitive processes to compensate for age related effects or pathology. Under the reserve hypothesis (Stern, 2002), education is being considered as one of the main proxy of reserve, although other factors (i.e. intelligence quotient (IQ), occupation, social and physical activities, complex mental activities) may also be considered (Satz et al., 2011). Functional studies during memory tasks showed functional reorganization of brain networks (compensation) in healthy elders with higher education compared to young individuals (Scarmeas et al., 2003), and more efficient or optimal patterns of brain activation in elders with higher reserve proxies compared to elders with lower reserve proxies (Bosch et al., 2010; Solé-Padullés et al., 2009). According to C. Bastin et al., 2012, for tasks of low to moderate difficulty, high cognitive reserve will take the form of reduced - more efficient activation of the network for an equivalent or even greater success in the task and for high-demanding tasks, individuals with high cognitive reserve will have a greater capacity, so that they can show greater increase in network activation to cope with increasing task difficulty.

With the insights gained from these studies we aimed to understand the coherence of positively and negatively correlated networks during a working memory task as we strongly believe that brain is better understood as a balanced network resonating in harmony during task performance. Education being a main proxy for cognitive reserve we also aimed at understanding the effect of education on the harmony of these networks.

\section{Materials and methodology}

\subsection{Sample}

Written informed consent was sought from all the participants as per the institute Ethics Committee. Sixty right handed educated healthy volunteers from both the genders ( 32 females and 28 males) in the age range of $18-40$ years were recruited and segregated into two groups of 30 each as school educated ( $\mathrm{SE}<10$ years of education) and college educated ( $C E>10$ years of education) on the basis of education acquired in years. School educated participants' inability to pursue further years of education was related to lack of opportunities and financial constraints due to socio economic status. The mean age of CE was $23.67 \pm 4.53$ years and SE was $26.06 \pm 5.49$ years was not statistically significant $(p<0.05)$. 
The mean number of years of education in CE group was $16.58 \pm 2.8$ years and SE was $7.93 \pm 2.34$ years.

\subsection{Magnetic resonance imaging (MRI) procedure and data acquisition}

Functional MRI (fMRI) images were acquired using a 3 Tesla scanner (Skyra, Siemens, Germany) with a 32 channel radio frequency coil. Patient head was restrained using foam pads to minimize head motion and to provide comfort. Pictures for the task were projected via stimulus presenting system E-sys and a projector screen with a mirror mounted on a head-coil. Functional images were acquired using a $\mathrm{T} 2{ }^{*}$-sensitive spin echo, echo planar imaging (EPI) sequence sensitive to blood oxygen level-dependent contrast. 165 dynamics were obtained applying the following EPI parameters: TR $3 \mathrm{~s}$, TE $.03 \mathrm{~s}$, FOV $192 \mathrm{~mm}$, slice thickness $4 \mathrm{~mm}$, number of slices 36 , voxel size $3 * 3 * 4 \mathrm{~mm}$ and the matrix $64 * 64$. After obtaining the fMRI images, T1-weighted, threedimensional, high-resolution imaging was performed to facilitate localization of fMRI activation. T1-MPRAGE was acquired in 176 slices in a single volume of TR: $1600 \mathrm{~ms}$ and TE: $0.35 \mathrm{~s}$, voxel size: $1^{*} 1^{*} 1 \mathrm{~mm}$ and all axial sections were oriented parallel to the anterior commissure-posterior commissure (AC-PC) line.

\section{3. fMRI experimental design}

The experimental design constituted a block design paradigm (rest-active), with each block including 10 measures for a period of $30 \mathrm{~s}$. The paradigm constituted 4 runs, each starting with rest block then encoding block, then rest block which was followed by retrieval block. Hence the paradigm consisted of sixteen blocks, four for encoding, four for retrieval and eight for rest. Eight rest blocks containing ten stimuli were presented prior to encoding and retrieval blocks in which four blocks were with + hair stimuli, four were with \# symbol. In the active period, four blocks were for encoding and remaining four blocks were for retrieval. The blocks were presented in a sequential manner. The paradigm started with + hair rest block followed by encoding block. In the encoding block, subjects were shown 10 pictures and were instructed to remember those. In the retrieval block also 10 pictures were presented, out of which 5 were repeated from the previous encoding block and 5 were new. Subjects were asked to identify those pictures what were repeated from the encoding block by a button press. The same sequences of blocks were repeated four times. There were a total of 20 pictures, 10 pictures for encoding item and 10 for retrieval item. In all the encoding blocks the same set of 10 pictures were presented but in a different order each time. In all the retrieval blocks 5 pictures were repeated from the previous encoding block and the remaining 5 were picked up from the item of retrieval list. So each block had a time period of $30 \mathrm{~s}$. The time gap between the encoding and retrieval blocks were $30 \mathrm{~s}$ ensuring the learning phase of the long term memory. A schematic diagram of experimental design included as Fig. 1. The visual stimuli (neutral outdoor scenes) were taken from the International affective picture system (Lang, Bradley, \& Cuthbert, 1999).

\section{Functional imaging data analysis}

\subsection{Preprocessing}

The functional and structural MRI pre analysis was performed using statistical parametric mapping (Friston, Ashburner, Kiebel, Nichols, \& Penny, 2011) (SPM8; Welcome Department of Cognitive Neurology, London). The data was realigned for motion correction by registration to the mean image. The images were then normalized to the Montreal Neurological Institute (MNI) space. Finally images were smoothed with a Gaussian kernel of $8 \mathrm{~mm}$ at fullwidth half maximum. In addition to these steps, the structural data was segmented for gray matter, white matter, and cerebrospinal fluid (CSF) areas for optional use during removal of temporal confounding factors, to remove the effect of head motion and for bias correction of magnetization in homogeneity.

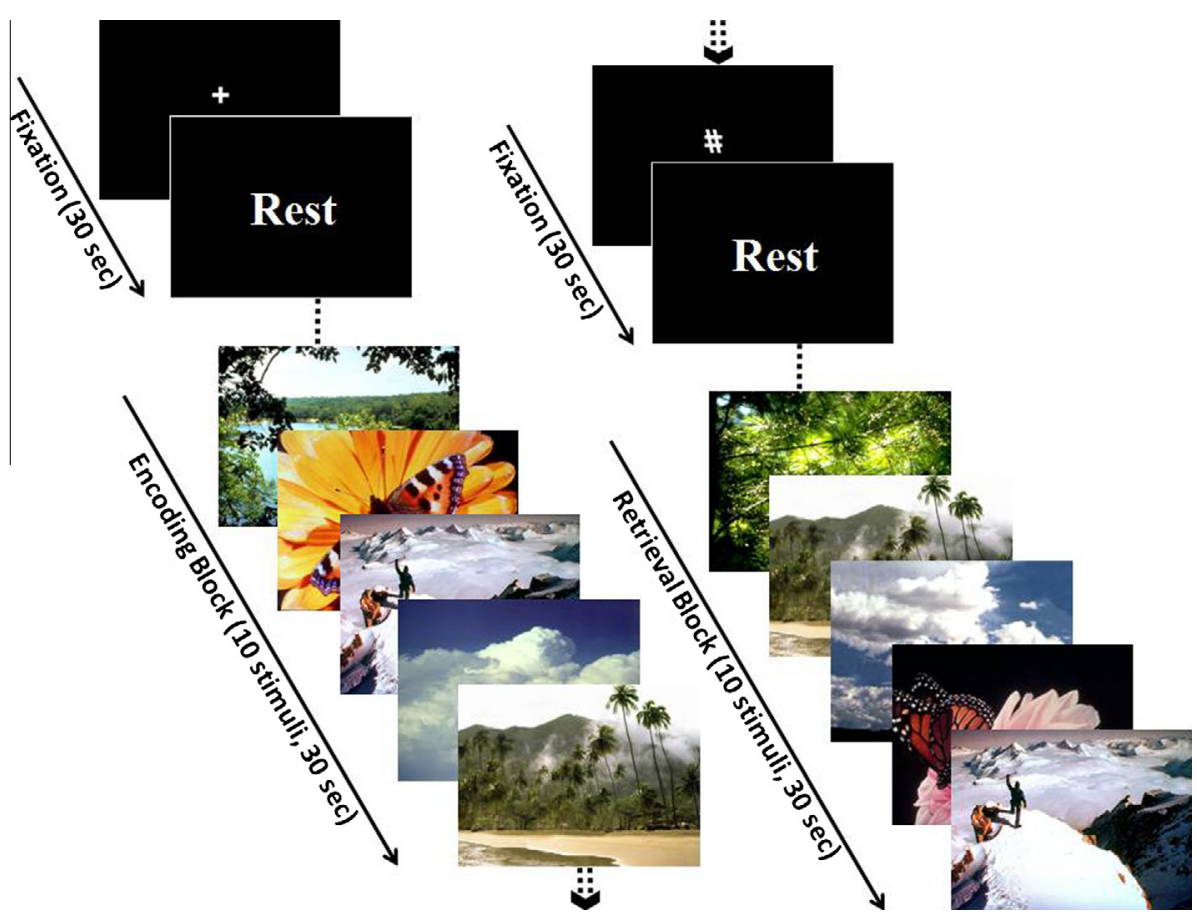

Fig. 1. Illustration of fMRI learning memory encoding and retrieval experimental design. 


\subsection{Functional connectivity analysis}

Seed to voxel based correlation approach was used to evaluate the temporally correlated blood oxygenation level dependent (BOLD) signal (Marquez de la Plata, Garces, Shokri Kojori, \& Diaz-Arrastia, 2011) associated with the functional connectivity of the brain networks. The fMRI functional Connectivity toolbox "CONN v.13.I" implemented in SPM (http://www.nitrc.org/projects/conn cited in Whitfield-Gabrieli \& Nieto-Castanon, 2012) was used.

The seed based connectivity performed by computing the temporal correlation between the BOLD signals from a given seed to all other voxels in the brain which used graph theoretical network analysis and topology of the cerebral network for analysis. The BOLD time series was extracted from each cluster in a network and correlated with the BOLD time-series signal of every other cluster in the network to create a correlation matrix showing connectivity between each region within the network. The realigned, normalized and smoothed images were taken for processing. White matter, CSF, physiological noise source reduction and gender distribution were taken as confounds, following the implemented CompCor strategy (Behzadi, Restom, Liau, \& Liu, 2007). The seed Region of interest (ROI) consisted of $6 \mathrm{~mm}$ radius spheres centered on MNI coordinates used to identify the corresponding networks. Bi-variate correlations were calculated between each pair of seed ROI and other brain region, as reflections of connections. All Brodmann areas were imported as possible connections for our selected seeds. The mean time series from each ROI was used as a predictor in a multiple regression General Linear Model (GLM) at each voxel. The PCC seed $(1,-55,17)$ was selected based on prior studies (Gordon, Breeden, Bean, \& Vaidya, 2012; Hampson et al., 2006; Sala-Llonch et al., 2012) to explore the exhibit and inhibitory effect during performance of memory task. The General Linear Model (GLM) was designed with Hann function to determine significant connections for each task (encoding and retrieval) (Whitfield-Gabrieli \& Nieto-Castanon, 2012) at the individual level for the CE group and SE group. Based upon 1st level, the second-level random-effect analysis was used to create within group statistical parametric maps (SPMs) for each network and to examine connectivity differences between groups. Three conditions were specified for encoding, retrieval and (baseline) rest period by defining the onset and duration and the activity for rest period was regressed out in the design matrix to determine task-related brain network connectivity. For the within group analyses, the SPMs generated for each network was given threshold at the wholebrain cluster-level (corrected alpha level .05; voxel-wise $p=0.001$ ) to show regions both positively and negatively correlated with the seed ROI. Between groups SPMs were thresholded at the whole-brain cluster-level (corrected alpha 0.05; voxel-wise $p$ value of 0.05 with False Discovery Rate (FDR) correction) (Woodward, Rogers, \& Heckers, 2011).

\section{Results}

\subsection{Behavioral results}

Performance scores ( $d$ prime) and reaction times (RTs) at the time of retrieval were noted for both the CE and SE group. All the subjects performed well (greater than $80 \%$ correct response) during the scanning session across the group. A Mann-Whitney $U$ test was used to evaluate their difference in behavioral preferences. CE showed significantly higher performance scores and shorter RTs in retrieval. $d$ Prime values are significantly different between the CE $(3.045 \pm 0.4917$, median $=3.16)$ and SE $(1.443 \pm 0.8265$, median $=1.61$ ) with $p$ value is less than 0.001 and $U$ is 31.5 . The mean of RTs is also significantly different between the CE
$(712 \pm 100.98 \mathrm{~ms}$, median $=711.5 \mathrm{~ms})$ and SE $(941.2 \pm 190.36 \mathrm{~ms}$, median $=874.5 \mathrm{~ms}$ ) with $p$ value is less than 0.001 and $U$ is 77.5 .

\subsection{Functional connectivity results}

Using the seed to voxel based correlation approaches, we analyzed the temporally correlated BOLD signal (Hampson, Peterson, Skudlarski, Gatenby, \& Gore, 2002; Marquez de la Plata et al., 2011) associated with functional connectivity for encoding and memory retrieval. The connection was identified at a significance level of $p<0.05$ with False Discovery Rate (FDR) correction. We focused on the region involved in DMN (PCC-R and MPFR) and DLPFR.

We considered the PCC region (PCC-R) as sum total cortex of ventral posterior cingulate cortex (VPCC), dorsal posterior cingulate cortex (DPCC), retrosplenial cingulate cortex (RCC) and PCC; MPFC region (MPFR) as sum total cortex of anterior prefrontal cortex (APFC), dorsal anterior cingulate cortex (DACC), anterior cingulate cortex (ACC) and MPFC; dorso-lateral pre frontal region (DLPFR) as sum total cortex of dorso-lateral pre frontal cortex (DLPFC), inferior frontal cortex (IFC), pars triangularis, IFC pars opercularis and dorsal frontal cortex (DFC).

\subsubsection{Results for encoding and retrieval}

Initially we studied connectivity across the two stages i.e., encoding and retrieval, irrespective of the study group. It was noted that the connectivity between bilateral PCC seed with other brain areas was higher during the process of retrieval as compared to encoding. For bilateral PCC seed, we noted that bilateral-inferior parietal cortex, MPFC and posterior cingulate (network known as DMN) were negatively correlated in both encoding and retrieval and there was strong negative correlation during encoding when compared to retrieval. While during retrieval, bilateral DLPFC, IFC pars triangularis, IFC pars opercularis, inferior prefrontal gyrus (IPFG) and supramarginal cortex were positively correlated. For encoding, bilateral DLPFC, R-IFC pars triangularis, R-IFC pars opercularis, bilateral supramarginal cortex and visual cortex were positively correlated (see Fig. 2a and b). To summarize, we observed that retrieval had greater number of positive associative networks as compared to encoding and lesser negative connectivity. On the other hand, encoding had greater number of negative associative networks as compared to retrieval and lesser positive connectivity.
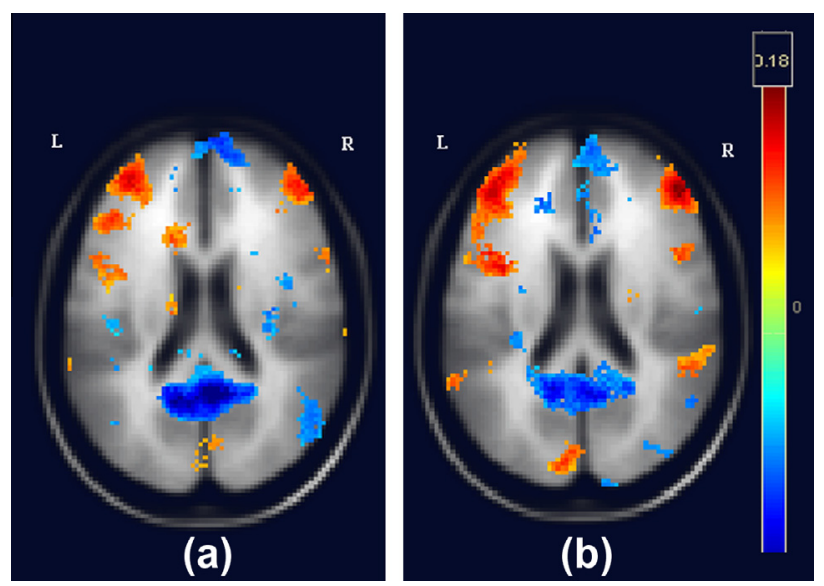

Fig. 2. Seed to voxel based connectivity for encoding (a) and retrieval (b) in posterior cingulate region seed. Red color shows significant positively correlated brain regions and blue color shows negatively correlated brain regions connected with source seed (posterior cingulate region) with the $p<0.05$ (FDR). (For interpretation of the references to color in this figure legend, the reader is referred to the web version of this article.) 

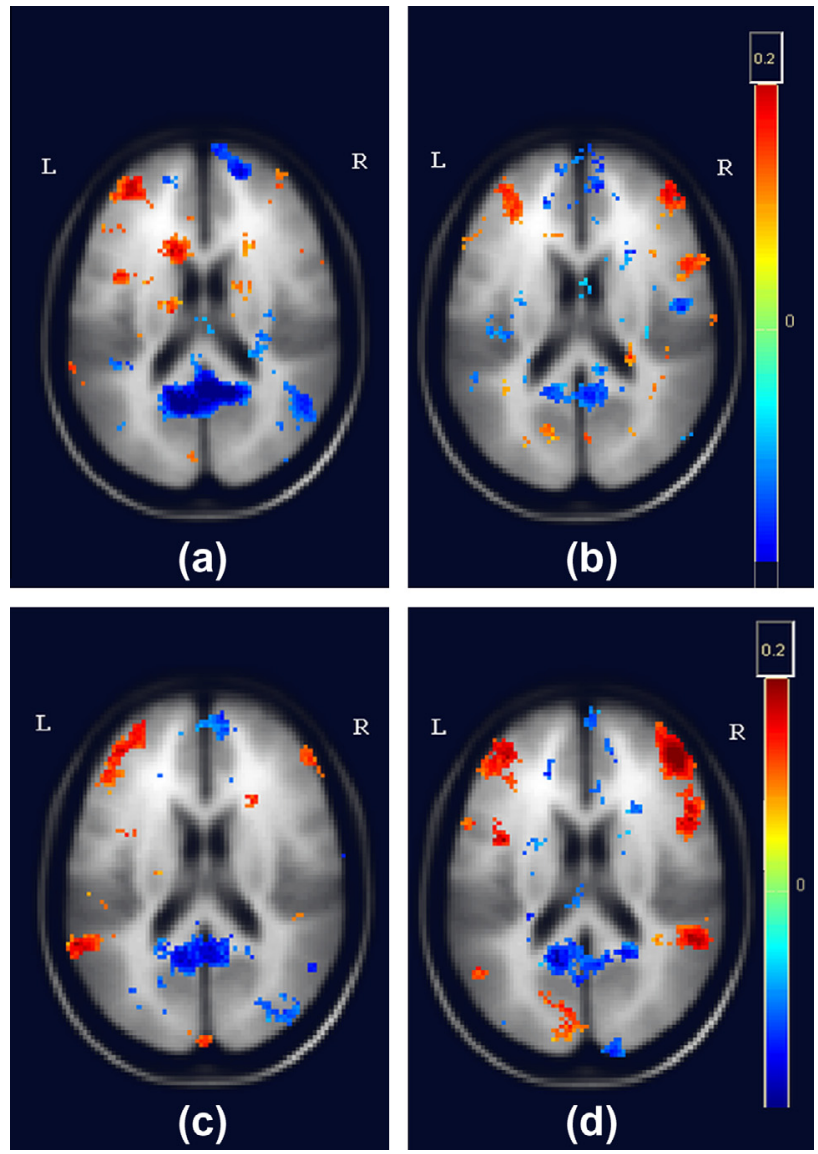

Fig. 3. Seed to voxel based connectivity for PCC seed for encoding and retrieval in the context of education. CE encoding (a), CE retrieval (b) SE encoding (c), SE retrieval (d). Red color demonstrating positive correlation with source seed and blue color demonstrating negative correlation with source seed. (For interpretation of the references to color in this figure legend, the reader is referred to the web version of this article.)

\subsubsection{Effect of education in encoding and retrieval}

Studies suggest that there is effective connectivity between PCC, MPFC and other brain areas which tend to show significant difference between the various study groups such as age and alcoholism (Esposito et al., 2009; Hampson et al., 2006; Sala-Llonch et al., 2012; Van den Bosch et al., 2012). We noted that, the PCC seeds showed significant difference in connectivity maps between college educated (CE) group and school educated (SE) group during both encoding and retrieval (see Fig. 3). Second level analyses revealed a significant pattern of functional connectivity between the CE and SE groups in the MPFR, PCC-R, DLPFR and supramarginal cortex. Table 1 shows the seed to voxel correlates of brain networks for PCC seeds, at $p<0.05$ (FDR corrected) and extent cluster threshold 0.005 scales. Beta represent Fisher-transformed correlation coefficient values ( $r$ is the correlation coefficient between the source seed area and correlated target voxel) ant $T$ is the strength of connection between the source seed area and correlated target voxel.

We noted that during encoding PCC seeds had higher negative correlation (activation with more voxel number, higher beta and $T$-values) with PCC-R and MPFR regions and lower positive correlation (activation with less voxel, lower beta and $T$-value) with bilateral DLPFR regions. The above mentioned negatively correlated networks were more negatively correlated at the time of encoding and the positive networks had more positive correlation at the time of retrieval. In the context of education as a baseline variable, it was noted that in the CE, both MPFC and PCC seeds had higher negative correlation with PCC-R and MPFR and lower positive correlation with bilateral DLPFR regions when compared to the SE both during encoding and retrieval (see Fig. 3).

\section{Discussion}

Brain regions simultaneously activated during any cognitive process are functionally connected, forming large-scale networks. These functional networks can be examined during task performance and rest states and such analysis is being widely used. The aim of our study was to examine brain networks connectivity for encoding and retrieval in the context of education. Sixty healthy young school educated and college educated subjects were selected to study the visual encoding and retrieval using fMRI. Using seed based connectivity; we explored the negative correlation of DMN and the positive correlation of dorsolateral frontal cortices, supramarginal gyrus.

Table 1

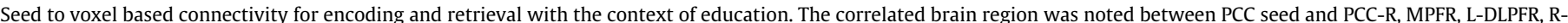

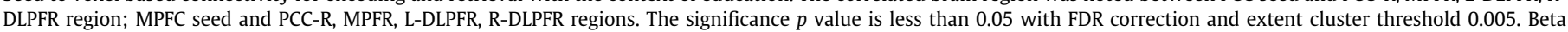

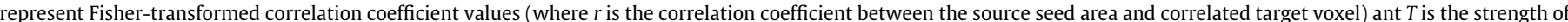

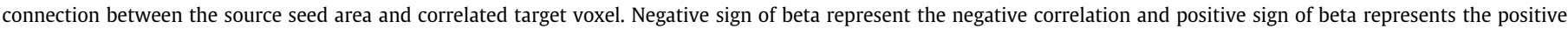

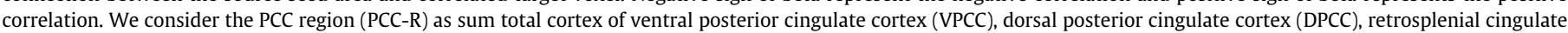

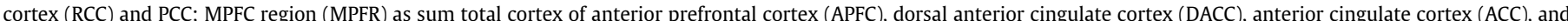

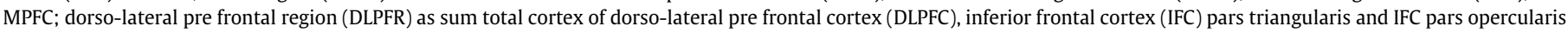
and dorsal frontal cortex (DFC).

\begin{tabular}{|c|c|c|c|c|c|c|c|c|c|}
\hline Condition & Source seed & Correlated region & $X$ & $Y$ & $Z$ & Cluster $(k)$ & Beta-value & $T$-value & P-FDR \\
\hline \multirow[t]{4}{*}{ CE_encoding } & \multirow[t]{4}{*}{ PCC } & PCC-R & -70 & -60 & 24 & 1382 & -.17 & 8.01 & 0000 \\
\hline & & MPFR & 07 & 58 & 02 & 272 & -.15 & 7.35 & 0000 \\
\hline & & L-DLPFR & -33 & 28 & 44 & 270 & .15 & 4.49 & 0001 \\
\hline & & R-DLPFR & 41 & 48 & 30 & 141 & .14 & 4.93 & 0000 \\
\hline \multirow[t]{4}{*}{ SE_encoding } & \multirow[t]{4}{*}{ PCC } & PCC-R & -30 & -48 & 46 & 376 & -.14 & 5.39 & 0000 \\
\hline & & MPFR & -13 & 34 & 04 & 181 & -.13 & 6.04 & 0052 \\
\hline & & L-DLPFR & -35 & 32 & 26 & 235 & .15 & 6.05 & 0000 \\
\hline & & R-DLPFR & 49 & 40 & 30 & 263 & .14 & 5.33 & 0001 \\
\hline \multirow[t]{4}{*}{ CE_retrieval } & \multirow[t]{4}{*}{ PCC } & PCC-R & -05 & -52 & 08 & 752 & -.16 & 7.24 & 0000 \\
\hline & & MPFR & 13 & 52 & 10 & 125 & -.15 & 5.15 & 0071 \\
\hline & & L-DLPFR & -35 & 44 & 10 & 425 & .15 & 6.00 & 0000 \\
\hline & & R-DLPFR & 55 & 44 & 18 & 113 & .12 & 5.71 & 0074 \\
\hline \multirow[t]{4}{*}{ SE_retrieval } & \multirow[t]{4}{*}{ PCC } & PCC-R & -09 & -52 & 18 & 325 & -.15 & 6.15 & 0000 \\
\hline & & MPFR & 12 & 52 & 11 & 67 & -.12 & 4.12 & 0082 \\
\hline & & L-DLPFR & -39 & 50 & 00 & 465 & .16 & 6.30 & 0007 \\
\hline & & R-DLPFR & 53 & 12 & 26 & 581 & .17 & 8.42 & 0000 \\
\hline
\end{tabular}


In our study of visual learning memory, a seed based connectivity analysis revealed that there is richer connection for retrieval than encoding with differential activations for encoding and retrieval. For PCC seed, we noted that the DMN networks were negatively correlated in both encoding and retrieval and there was stronger negative correlation during encoding as compared to retrieval. This represents the brain facilitation during attentive task performance at the time of encoding rather than retrieval. Positive correlations were observed in DLPFR, bilateral inferior prefrontal gyrus and supramarginal cortex and were stronger at the time of retrieval rather than encoding which suggest that there are auxiliary planning, executive and information correlation at the time of retrieval. The visual networks were positively correlated during encoding and negatively correlated during retrieval. The increase in the number of connections during retrieval could be due to the fact that the neural mechanisms of the processes overlap and hence possibly while retrieving the information, the healthy volunteers could have used the information about the way they have encoded the information. This is supported by the theory of encoding specificity principle, (Tulving, 1995), transfer appropriate processing (Morris, Bransford, \& Franks, 1977).

Retrieval processes when explored point involvement of dorsolateral/ventrolateral prefrontal cortex (DLPFC/VLPFC) and medial temporal lobe (MTL) structures (Buckner, Koutstaal, Schacter, Wagner, \& Rosen, 1998; Buckner, Raichle, Miezin, \& Petersen, 1996). Verbal material activates left lateralized VLPFC/DLPFC and left medial temporal structures (Kelley et al., 1998). Prince, Daselaar, and Cabeza (2005) reported that cortical activity related to encoding and retrieval was associated with MTL structures (encoding = anterior hippocampus; retrieval = posterior hippocampus/parahippocampal gyrus) as well as with prefrontal cortices (encoding $=$ VLPFC; retrieval $=$ DLPFC and anterior PFC). One of the study suggested that encoding activations tended to be clustered in anterior MTL regions and retrieval in more posterior MTL (hippocampal encoding/retrieval pattern or "HIPER") regions (Lepage, Habib, \& Tulving, 1998). It was predicted that activation in ventral posterior parietal and dorsolateral prefrontal cortex in perceptual identification priming for encoding, whereas activation in ventrolateral prefrontal and dorsal posterior parietal cortex for recognition memory.

We hypothesized that education is an important factor which could possibly alter the cognitive networks thus influencing the performance or vice versa. There was significant difference between SE and CE both in terms of their performance and brain regions activated. The performance of the $\mathrm{CE}$ was better than the $\mathrm{SE}$ as evidenced by better mean $d$ prime score and faster RTs. The brain areas activated suggest that there was a higher negative correlation for CE when compared to SE however the positive correlations were greater for SE than CE. SE has primarily used DLPFR when compared to CE. The DLPFC is the core structure for higher order executive functions and encoding (Stuss \& Levine, 2002). The performance of the CE is higher when compared to SE and relatively their brain activations for DLPFR are lesser indicative of the possibility that the CE could have used cognitive efficiency principle. Faster performers showed less prefrontal cortical activity than slower performers and in parietal regions they showed greater activity. PFC exerted more influence over other brain regions for slower than for faster individuals when Regional-causality analysis was performed. The results suggest that a critical determinant of individual performance differences is the efficiency of interactions between brain regions and that slower individual may require more prefrontal executive control than faster individuals to perform successfully (Rypma et al., 2006). In another study, left inferior parietal lobule was strongly connected with the left hippocampus during encoding, resulting increase memorial content (increased numbers of associations to be bound). It was hypothesized that the onset of strategic processing led to a suppression of the lateral parietal attentional mechanisms, resulting in negative connectivity and prefrontal and hippocampal connection regions which are critically involved in potentially dissociable aspects of successful encoding serves as a strong impetus to begin evaluating how these regions interact with each other and within a broader network (Addis \& McAndrews, 2006).

In summary we found that there were reciprocal dynamic changes in both DLPFC and PCC regions during working memory encoding and retrieval. In agreement with the previous studies, there were more positively correlated regions during retrieval compared to encoding and DMN networks showed greater deactivations during more attentive task of visual encoding. There were significant differences in functional connectivity between the college educated and school educated group and education seems to have influenced both encoding and retrieval. College educated had relatively lesser positively correlated DLPFC regions and higher negatively correlated PCC regions indicative of the possibility that the CE could have used cognitive efficiency principle. The limitation of our study is that we could investigate only the measure of educational attainment, a major component of cognitive reserve and not other important components in reserve such as occupation, intelligence quotient, socio economic status and complex mental activities. Future studies could be directed to understand the effect of these factors on cognitive networks. Longitudinal studies also could explore the changes in the networks with varying levels of education. Such data would help us to monitor cognitive rehabilitation and to develop tailor made cognitive tasks for patients focusing on various parameters such as level of education, tasks, efficiency and cognitive load.

\section{Acknowledgments}

This research work was funded by the Department of Science and Technology. We thank our radiographers, (Dept. Neuroimaging and Interventional Radiology (NIIR), NIMHANS, India) for their technical support and data collection. Special thanks to Mr. Deepak Ulal (Senior Technician, Dept. of Clinical Psychology) and Ms. Saranya VR (Radiographer, Dept. NIIR).

\section{References}

Addis, D. R., \& McAndrews, M. P. (2006). Prefrontal and hippocampal contributions to the generation and binding of semantic associations during successful encoding. Neuroimage, 33(4), 1194-1206.

Bastin, C., Yakushev, I., Bahri, M. A., Fellgiebel, A., Eustache, F., Landeau, B., \& Salmon, E. (2012). Cognitive reserve impacts on inter-individual variability in restingstate cerebral metabolism in normal aging. NeuroImage, 63(2), 713-722.

Behzadi, Y., Restom, K., Liau, J., \& Liu, T. T. (2007). A component based noise correction method (CompCor) for BOLD and perfusion based fMRI. Neuroimage, 37(1), 90-101.

Bjork, R. A., \& Whitten, W. B. (1974). Recency-sensitive retrieval processes in longterm free recall. Cognitive Psychology, 6(2), 173-189.

Bosch, B., Bartrés-Faz, D., Rami, L., Arenaza-Urquijo, E. M., Fernández-Espejo, D. Junqué, C., \& Molinuevo, J. L. (2010). Cognitive reserve modulates task-induced activations and deactivations in healthy elders, amnestic mild cognitive impairment and mild Alzheimer's disease. Cortex, 46(4), 451-461.

Buckner, R. L., Andrews-Hanna, J. R., \& Schacter, D. L. (2008). The brain's default network. Annals of the New York Academy of Sciences, 1124(1), 1-38.

Buckner, R. L., Koutstaal, W., Schacter, D. L., Wagner, A. D., \& Rosen, B. R. (1998) Functional-anatomic study of episodic retrieval using fMRI. Neuroimage, 7(3), 151-162.

Buckner, R. L., Raichle, M. E., Miezin, F. M., \& Petersen, S. E. (1996). Functional anatomic studies of memory retrieval for auditory words and visual pictures. The Journal of Neuroscience, 16(19), 6219-6235.

Champod, A. S., \& Petrides, M. (2007). Dissociable roles of the posterior parietal and the prefrontal cortex in manipulation and monitoring processes. Proceedings of the National Academy of Sciences, 104(37), 14837-14842.

Champod, A. S., \& Petrides, M. (2010). Dissociation within the frontoparietal network in verbal working memory: A parametric functional magnetic resonance imaging study. The Journal of Neuroscience, 30(10), 3849-3856. 
Chanraud, S., Pitel, A., Pfefferbaum, A., \& Sullivan, E. V. (2011). Disruption of functional connectivity of the default-mode network in alcoholism. Cerebral Cortex, 10(1093), 1-12.

Collette, F., Salmon, E., Van der Linden, M., Chicherio, C., Belleville, S., Degueldre, C., et al. (1999). Regional brain activity during tasks devoted to the central executive of working memory. Cognitive Brain Research, 7(3), 411-417.

Craik, F. I., \& Tulving, E. (1975). Depth of processing and the retention of words in episodic memory. Journal of Experimental Psychology: General, 104(3), 268-294.

Damoiseaux, J. S., Rombouts, S. A. R. B., Barkhof, F., Scheltens, P., Stam, C. J., Smith, Stephen M., \& Beckmann, C. F. (2006). Consistent resting-state networks across healthy subjects. Proceedings of the national academy of sciences, 103(37), 13848-13853.

Deiber, M. P., Wise, S. P., Honda, M., Catalan, M. J., Grafman, J., \& Hallett, M. (1997). Frontal and parietal networks for conditional motor learning: A positron emission tomography study. Journal of Neurophysiology, 78(2), 977-991.

Eichenbaum, H. (2011). The cognitive neuroscience of memory: An introduction. OUP USA.

Esposito, F., Aragri, A., Latorre, V., Popolizio, T., Scarabino, T., Cirillo, S., et al. (2009). Does the default-mode functional connectivity of the brain correlate with working-memory performances? Archives Italiennes de Biologie, 147(1/2), $11-20$.

Fox, M. D., \& Raichle, M. E. (2007). Spontaneous fluctuations in brain activity observed with functional magnetic resonance imaging. Nature Reviews Neuroscience, 8, 700-711.

Fox, M. D., Snyder, A. Z., Vincent, J. L., Corbetta, M., Van Essen, D. C., \& Raichle, M. E (2005). The human brain is intrinsically organized into dynamic, anticorrelated functional networks. Proceedings of the National Academy of Sciences of the United States of America, 102(27), 9673-9678.

Fox, M. D., Zhang, D., Snyder, A. Z., \& Raichle, M. E. (2009). The global signal and observed anticorrelated resting state networks. Journal of Neurophysiology, 101(6), 3270-3283.

Fransson, A. (2005). Spontaneous low-frequency BOLD signal fluctuations: An fMR investigation of the resting-state default mode of brain function hypothesis Human Brain Mapping, 26(1), 15-29.

Friston, K. J., Ashburner, J. T., Kiebel, S. J., Nichols, T. E., \& Penny, W. D. (2011). Statistical parametric mapping: The analysis of functional brain images: The analysis of functional brain images. Academic Press.

Fukushima, T., Hasegawa, I., \& Miyashita, Y. (2003). Prefrontal neuronal activity encodes spatial target representations sequentially updated following nonspatial target-shift cues. J Neurophysiol, 91, 1367-1380.

Fuster, J. M. (1997). Network memory. Trends in neurosciences, 20(10), 451-459.

Gerton, B. K., Brown, T. T., Meyer-Lindenberg, A., Kohn, P., Holt, J. L., Olsen, R. K. et al. (2004). Shared and distinct neurophysiological components of the digits forward and backward tasks as revealed by functional neuroimaging. Neuropsychologia, 42(13), 1781-1787.

Gordon, M. E., Breeden, A. L., Bean, S. E., \& Vaidya, C. J. (2012). Working memoryrelated changes in functional connectivity persist beyond task disengagement. Human Brain Mapping, 1-14.

Greicius, M. D., Krasnow, B., Reiss, A. L., \& Menon, V. (2003). Functional connectivity in the resting brain: A network analysis of the default mode hypothesis. Proceedings of the National Academy of Sciences, 100(1), 253-258.

Greicius, M. D., Supekar, K., Menon, V., \& Dougherty, R. F. (2009). Resting-state functional connectivity reflects structural connectivity in the default mode network. Cerebral Cortex, 19(1), 72-78.

Hampson, M., Driesen, N. R., Skudlarski, P., Gore, J. C., \& Constable, R. T. (2006). Brain connectivity related to working memory performance. The Journal of Neuroscience, 26(51), 13338-13343.

Hampson, M., Peterson, B. S., Skudlarski, P., Gatenby, J. C., \& Gore, J. C. (2002) Detection of functional connectivity using temporal correlations in MR images. Human Brain Mapping, 15(4), 247-262.

Iidaka, T., Matsumoto, A., Nogawa, J., Yamamoto, Y., \& Sadato, N. (2006). Frontoparietal network involved in successful retrieval from episodic memory. Spatial and temporal analyses using fMRI and ERP. Cerebral Cortex, 16(9), 1349-1360.

Johnson, B., Zhang, K., Gay, M., Horovitz, S., Hallett, M., Sebastianelli, W., et al. (2012). Alteration of brain default network in subacute phase of injury in concussed individuals: Resting-state fMRI study. Neuroimage, 59(1), 511-518.

Kelley, W. M., Miezin, F. M., McDermott, K. B., Buckner, R. L., Raichle, M. E., Cohen, N. J., et al. (1998). Hemispheric specialization in human dorsal frontal cortex and medial temporal lobe for verbal and nonverbal memory encoding. NeuronCambridge MA-, 20, 927-936.

LaBar, K. S., Gitelman, D. R., Parrish, T. B., \& Mesulam, M. (1999). Neuroanatomic overlap of working memory and spatial attention networks: A functional MRI comparison within subjects. Neuroimage, 10(6), 695-704.
Lang, P. J., Bradley, M. M., \& Cuthbert, B. N. (1999). International affective picture system (IAPS): Technical manual and affective ratings. Gainesville, FL: The Center for Research in Psychophysiology, University of Florida.

Lepage, M., Habib, R., \& Tulving, E. (1998). Hippocampal PET activations of memory encoding and retrieval: The HIPER model. Hippocampus, 8(4), 313-322.

Marquez de la Plata, C., Garces, J., Shokri Kojori, E., \& Diaz-Arrastia, R. (2011). Deficits in functional connectivity of hippocampal and frontal lobe circuits after traumatic diffuse axonal Injury. Archives of Neurology, 68(1), 74-84.

Mayer, J. S., Roebroeck, A., Maurer, K., \& Linden, D. E. (2010). Specialization in the default mode: Task-induced brain deactivations dissociate between visual working memory and attention. Human Brain Mapping, 31(1), 126-139.

Meda, S. A., Stevens, M. C., Folley, B. S., Calhoun, V. D., \& Pearlson, G. D. (2009). Evidence for anomalous network connectivity during working memory encoding in schizophrenia: An ICA based analysis. PLoS One, 4(11), e7911.

Michels, L., Bucher, K., Lüchinger, R., Klaver, P., Martin, E., Jeanmonod, D., et al. (2010). Simultaneous EEG-fMRI during a working memory task: Modulations in low and high frequency bands. PLoS One, 5(4), e10298.

Milner, B., Squire, L. R., \& Kandel, E. R. (1998). Cognitive neuroscience review and the study of memory. Neuron, 20, 445-468.

Morris, C. D., Bransford, J. D., \& Franks, J. J. (1977). Levels of processing versus transfer appropriate processing. Journal of Verbal Learning and Verbal Behavior, 16(5), 519-533.

Prince, S. E., Daselaar, S. M., \& Cabeza, R. (2005). Neural correlates of relational memory: Successful encoding and retrieval of semantic and perceptual associations. The Journal of Neuroscience, 25(5), 1203-1210.

Raichle, M. E., MacLeod, A. M., Snyder, A. Z., Powers, W. J., Gusnard, D. A., \& Shulman, G. L. (2001). A default mode of brain function. Proceedings of the National Academy of Sciences, 98(2), 676-682.

Rypma, B., Berger, J. S., Prabhakaran, V., Martin Bly, B., Kimberg, D. Y., Biswal, B. B., et al. (2006). Neural correlates of cognitive efficiency. Neuroimage, 33(3), 969-979.

Sala-Llonch, R., Peña-Gómez, C., Arenaza-Urquijo, E. M., Vidal-Piñeiro, D., Bargalló, N., Junqué, C., et al. (2012). Brain connectivity during resting state and subsequent working memory task predicts behavioural performance. Cortex, 48(9), 1187-1196.

Satz, P., Cole, M. A., Hardy, D. J., \& Rassovsky, Y. (2011). Brain and cognitive reserve: Mediator (s) and construct validity, a critique. Journal of Clinical and Experimental Neuropsychology, 33(1), 121-130.

Scarmeas, N., \& Stern, Y. (2003). Cognitive reserve and lifestyle. Journal of clinical and experimental neuropsychology, 25(5), 625-633.

Solé-Padullés, C., Bartrés-Faz, D., Junqué, C., Vendrell, P., Rami, L., Clemente, I. C., \& Molinuevo, J. L. (2009). Brain structure and function related to cognitive reserve variables in normal aging, mild cognitive impairment and Alzheimer's disease. Neurobiology of aging, 30(7), 1114-1124.

Stern, Y. (2002). What is cognitive reserve? Theory and research application of the reserve concept. Journal of the International Neuropsychological Society, 8(03), $448-460$.

Stuss, D. T., \& Levine, B. (2002). Adult clinical neuropsychology: Lessons from studies of the frontal lobes. Annual Review of Psychology, 53(1), 401-433.

Sun, X., Zhang, X., Chen, X., Zhang, P., Bao, M., Zhang, D., et al. (2005). Agedependent brain activation during forward and backward digit recall revealed by fMRI. Neuroimage, 26(1), 36-47.

Tomasi, D., Ernst, T., Caparelli, E. C., \& Chang, L. (2006). Common deactivation patterns during working memory and visual attention tasks: An intra-subject fMRI study at 4 Tesla. Human Brain Mapping, 27(8), 694-705.

Tulving, E. (1995). Organization of memory: Quo vadis. The Cognitive Neurosciences, 839-847.

Uddin, L. Q., Clare Kelly, A. M., Biswal, B. B., Castellanos, F. X., \& Milham, M. P. (2009). Functional connectivity of default mode network components: Correlation, anticorrelation, and causality. Human Brain Mapping, 30(2), 625-637.

Van den Bosch, G. E., Marroun, H. E., Schmidt, M. N., Tibboel, D., Manoach, D. S. Calhoun, V. D., et al. (2012). Brain connectivity during verbal working memory in children and adolescents. Human Brain Mapping.

Wang, J. X., Bartolotti, J., Amaral, L. A., \& Booth, J. R. (2013). Changes in task-related functional connectivity across multiple spatial scales are related to reading performance. PLoS One, 8(3), e59204.

Whitfield-Gabrieli, S., \& Nieto-Castanon, A. (2012). Conn: A functional connectivity toolbox for correlated and anticorrelated brain networks. Brain Connectivity, 2(3), 125-141.

Woodward, N. D., Rogers, B., \& Heckers, S. (2011). Functional resting-state networks are differentially affected in schizophrenia. Schizophrenia Research, 130(1), 86-93. 